\title{
Kernos
}

Revue internationale et pluridisciplinaire de religion grecque antique

32 | 2019

Varia

\section{Géographie, paléographie et philologie.}

Note sur un lieu de culte de Déméter

\section{Denis Rousset}

\section{Q OpenEdition}

1 Journals

Édition électronique

URL : https://journals.openedition.org/kernos/3141

DOI : $10.4000 /$ kernos.3141

ISSN : 2034-7871

Éditeur

Centre international d'étude de la religion grecque antique

Édition imprimée

Date de publication : 1 décembre 2019

Pagination : 237-240

ISBN : 978-2-87562-229-7

ISSN : 0776-3824

Référence électronique

Denis Rousset, « Géographie, paléographie et philologie. », Kernos [En ligne], 32 | 2019, mis en ligne le 01 octobre 2021, consulté le 31 janvier 2022. URL : http://journals.openedition.org/kernos/3141 ; DOI https://doi.org/10.4000/kernos.3141

Ce document a été généré automatiquement le 31 janvier 2022.

Kernos 


\title{
Géographie, paléographie et philologie.
}

\author{
Note sur un lieu de culte de Déméter
}

Denis Rousset

1 Dans quelle mesure peut-on retoucher le texte transmis et admis d'une œuvre ancienne à la lumière des découvertes de terrain ? C'est la question que suscite un récent article de M. Tiverios qui, aidé par Chr. Tsagalis, a proposé de substituer, dans le vers 491 de l'Hymne homérique à Déméter, à l'obscure cité d'Achaïe Phthiotide Antrôn la célèbre et cycladique Andros ${ }^{1}$.

2 Rappelons que l'hymne se clôt (v. 490-495) par un appel adressé à Déméter et Perséphone, dont le texte reçu commence ainsi :

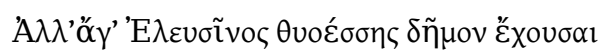

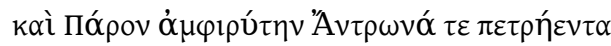

Hé bien! vous qui possédez la terre odorante d'Éleusis, et Paros ceinte par les flots,

et la rocheuse Antrôn, etc. ${ }^{2}$.

3 Alors que Paros est bien connue pour le culte de Déméter ${ }^{3}$, Antrôn (Av $v \rho \omega ́ v$ ou

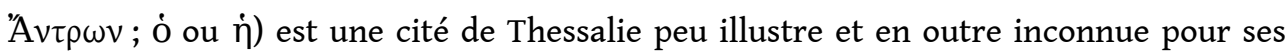
cultes. Aussi les commentateurs de l'Hymne n'ont-ils guère réussi à éclairer sa mention dans le poème que grâce à son voisinage, dans le livre II de l'Iliade, avec Pyrasos, lieu

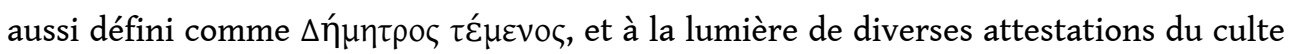
de Déméter en Thessalie ${ }^{4}$.

4 C'est un autre cheminement qui a conduit récemment $\mathrm{M}$. Tiverios à ce vers de l'Hymne. En effet, l'archéologue et historien a dressé pour l'île d'Andros un riche tableau synthétique, que nourrissent de nombreuses sources de tous ordres (topographie, épigraphie, numismatique, iconographie), du culte de Déméter, qui est attesté du haut archaïsme à l'époque hellénistique. C'est en effet à une haute époque que remonte le culte de la déesse à Andros, puisqu'y a été exhumé au site aujourd'hui appelé $Y \psi \eta \lambda \eta n$ un sanctuaire de la fin de l'époque géométrique, portant un temple d'époque archaïque et sans doute un Thesmophorion ${ }^{5}$. Aussi M. Tiverios oppose-t-il au silence des sources sur Déméter à Antrôn de Phthiotide l'ancienneté et la vigueur du culte de la divinité à 
Andros, et il s'étonne qu'un culte aussi précoce et vif ne soit pas cité de pair avec ceux d'Éleusis et de Paros dans l'Hymne, texte rédigé vers la première moitié du vi ${ }^{e}$ siècle ${ }^{6}$.

Considérant qu'il existe ainsi entre le texte et le terrain une divergence flagrante, M. Tiverios en vient à mettre en question le texte transmis et reçu de l'Hymne, lequel est parvenu jusqu'à nous grâce à un manuscrit unique, le Leidensis B.P.G. 33H, tardif $\left(\mathrm{xv}^{\mathrm{e}}\right.$ siècle) et non dépourvu de fautes ${ }^{7}$. Ainsi, n'y aurait-il donc pas également une erreur dans le texte du vers 491 ? Il faudrait y lire, non pas

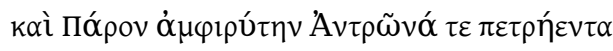

mais

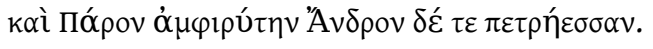

Cependant, au cours de la transmission du texte, on aurait, selon M. Tiverios, remplacé 'A $\delta \delta \rho o v$ par A A $\tau \rho \tilde{\omega} v \alpha$ : le « copiste » aurait en effet «corrigé le manuscrit », parce que d'une part il prenait en considération la mention de la cité thessalienne dans l'Iliade peu

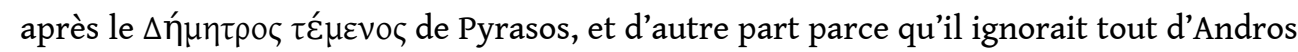
et d'un culte de Déméter dans l'île cycladique à l'époque "homérique». Ainsi, à la

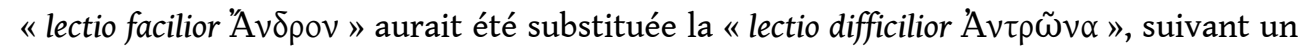
processus paradoxal relevé par M. Tiverios, qui souligne pour finir le fait que la mention originelle d'Andros dans l'Hymne, non seulement s'accorderait avec les sources de terrain, mais aussi ferait remonter la première citation écrite de l'île d'environ un siècle par rapport à sa mention chez Eschyle (Perses, 887) ${ }^{8}$.

Comment cependant expliquer la transformation du vers? C'est ici que l'historienarchéologue appelle à la rescousse un philologue, chargé d'expliquer le processus qui aboutit à l'erreur depuis toujours reçue, mais enfin reconnue et corrigée. Selon $\mathrm{Chr}$. Tsagalis, il faudrait distinguer trois phases :

- Le texte aurait présenté aux «1stc. B.C. - 2nd c. $A D$ » KAIחAPONAM d'autre part le vers aurait, d'après l'avis ici divergent de M. Chr. Tsagalis, présenté l'adjectif au masculin, qui s'appliquait à 'A?????, toponyme pourtant f?minin.

$v \delta \rho \circ \zeta$, toponyme pourtant féminin.

- Un copiste postérieur aurait méconnu le signe suivant le $\Delta$ et d'autre part confondu, dans l'écriture en onciales, cette dernière lettre avec un $\mathrm{A}$, écrivant donc pour sa part AN $\triangle$ PONATE, sans cependant comprendre le vers.

- Enfin, un autre copiste «with some philological background» entreprit de rendre

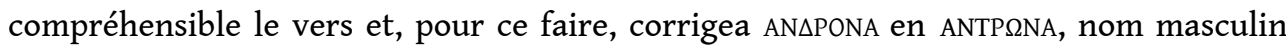
qui s'accordait avec l'adjectif.

9 Résumer l'explication en montre la faiblesse. Qu'il suffise des remarques suivantes :

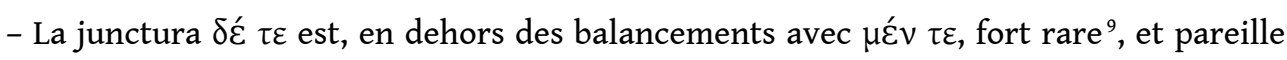
coordination serait, dans le vers supposé originel, tout à fait inutile, sauf en tant que cheville métrique, qui vient combler le manque d'un pied provoqué par la correction proposée.

- Si la substitution d'ANAPONA à ANAPON $\triangle$ peut remonter à la phase où le texte était en onciales, comment en revanche concevoir que le texte ait présenté à un certain moment $\Delta^{\prime}$, c'est-à-dire qu'il y ait eu, sinon une impossible élision, du moins une abréviation de $\delta \varepsilon$, ou une haplographie, alors que la lettre supposée suivre alors immédiatement l'E disparaissant, à savoir un $\mathrm{T}$, a une forme bien différente d'un $\mathrm{E}^{10}$ ? 
En outre, peut-on admettre qu'un copiste ait écrit, dans une phase quelconque de la transmission, la séquence $A N \triangle P O N \Delta^{\prime} T E$, inadmissible pour la métrique?

- Si à l'origine le vers portait en sa fin la forme $\pi \varepsilon \tau \rho \eta ́ \varepsilon \sigma \sigma \alpha v$, comme le propose pour sa part M. Tiverios, comment expliquer le passage à $\pi \varepsilon \tau p \eta ́ \varepsilon v \tau \alpha$ ? Quant à supposer avec

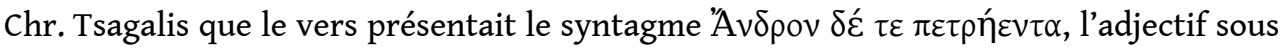
cette forme qualifiant le nom féminin de l'île, comme il arrive sans doute pour un autre

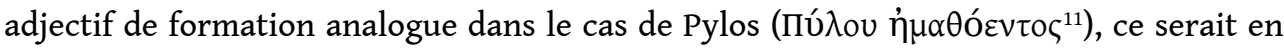
l'occurrence bien singulier, puisque c'est bien $\pi \varepsilon \tau \rho \eta ́ \varepsilon \sigma \sigma \alpha$ qui qualifie, de façon attendue, des noms féminins chez Homère, qui plus est à cette position dans l'hexamètre $^{12}$.

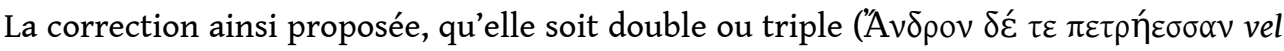

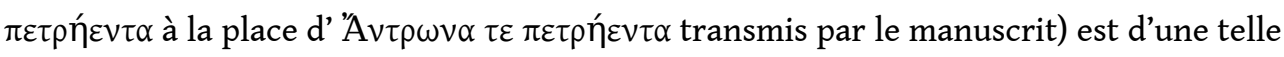
témérité, et l'histoire du texte ainsi reconstituée d'une telle invraisemblance qu'elles ne peuvent être acceptées. Quel que doive être le désir du géographe et philologue d'accorder la «terre » et le "papier ${ }^{13}$, l'histoire philologique et paléographique d'un texte ne peut être ainsi dictée par la carte des cultes matériellement attestés. Car, à cette aune-là, combien de passages de la littérature grecque ne pourrait-on retoucher à la lumière des explorations archéologiques et de leurs avancées, cependant non moins provisoires qu'aléatoires? En l'état actuel de l'histoire du texte de l'Hymne et de celle de la cité thessalienne, mieux vaut sans doute faire retour à la source textuelle telle qu'elle nous est parvenue et s'abstenir à la fois de ressusciter Andros dans le poème du $\mathrm{VI}^{\mathrm{e}}$ siècle et de priver la si chétive Antrôn du culte de Déméter.

\section{NOTES}

1. M. TIVERIOS, "The Cult of Demeter on Andros and the Homeric Hymn to Demeter ", Trends in Classics 9 (2017), p. 71-84 (p. 81-82: Appendix par Chr. TSAGALIS). Sur cette publication j'ai eu l'attention attirée lors de l'une des passionnantes conférences que Mme Chr. Mitsopoulou a données sur l'archéologie et les cultes éleusiniens en novembre 2018 au Collège de France, à l'invitation de notre collègue et amie Mme V. Pirenne-Delforge.

2. Texte et trad. (légèrement modifiée) J. Humbert, Collection des Universités de France, 1936, p. 58.

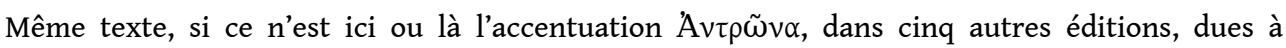
T.W. Allen, W.R. Hallyday et E.E. Sikes, N.J. Richardson, F. Càssola, M.L. West, A. Bernabé.

3. Pour Déméter à Paros, voir TIVERIOS, o.c., p. 78 et n. 19.

4. Sur Antrôn en Achaïe Phthiotide, voir, outre J.-Cl. DECOURT, Th. NIELSEN, B. HELLY, dans M.H. HANSEN, Th. HEINE NIELSEN (éd.), An inventory of archaic and classical poleis, Oxford, 2004, p. 713 $\mathrm{n}^{\circ}$ 433, M. BILLERBECK dans l'édition de Stéphane de Byzance (Berlin, 2004, s.v.), et G. zACHOS, Tabula imperii romani. J 34-Athens. Achaia Phthiotis-Malis-Aenis-Oitaia-Doris-Eurytania-East \& West LocrisPhokis-Aetolia-Akarnania, Athens, 2016, p. 22-23, plusieurs articles de M.-Ph. PAPAKonstantinou

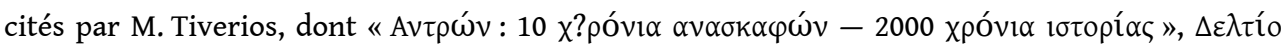

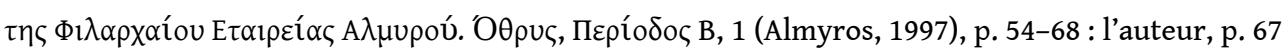
n. 3 et 4 , d'une part souligne qu'une partie du site urbain a pu depuis l'Antiquité disparaître dans 
la mer et d'autre part voit dans les nombreuses grottes en partie encore visibles sur place le lieu possible du culte de Déméter. - Antrôn est citée dans le Catalogue de l'Iliade peu après Pyrasos, Iliade II, 695-697.

5. Voir TIVERIOS, o.c., p. 71-77, avec les références utiles.

6. Sur la date admise pour la rédaction de l'Hymne, la première moitié $\mathrm{du} \mathrm{VI}^{\mathrm{e}} \mathrm{s}$., cf. l'édition de N.J. RICHARDSON, The Homeric Hymn to Demeter, Oxford, 1974, p. 5-11, et M.L. WEST, Homeric Hymns, Cambridge, 2003, p. 9.

7. Cf. récemment Chr. SIMELIDIS, "On the Homeric Hymns in Byzantium ", in A. FAULKNER, A. Vergados et A. schwab (éd.), The Reception of the Homeric Hymns, Oxford, 2016, p. 242-260, ici p. $252-260$.

8. TIVERIOS, o.c. p. 79-81, qui écrit cependant dans la même page : «why [...] was the lectio facilior 'A $v \delta \rho \circ v$ changed into the lectio difficilior A $A \tau \rho \tilde{\omega} v \alpha$ ? " et " the lectio facilior 'A $v \delta \rho o v$ replaced the

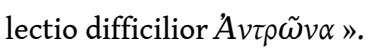

9. Voir J.D. DENNISTON, The Greek Particles, $2^{e}$ éd. Oxford, 1953, p. 529-532.

10. Je remercie Ph. Hoffmann et B. Mondrain pour les assurances et précisions qu'ils m'ont apportées dans la discussion de cette question paléographique.

11. Sur le nom Пú̉os dans l'épopée, voir cependant Lexikon des frühgriechischen Epos 20 (2004), s.v. col. 1645, qui admet les deux genres ; cf. aussi RE XXIII (1959), s.v. col. 2113-2114.

12. Voir Lexikon des frühgriechischen Epos 12 (1987), 909, et 20 (2004), 1200-1201, s. v.

13. Je fais allusion à l'œuvre de L. Robert, et notamment à la fameuse conférence « Géographie et philologie ou la Terre et le Papier ", Actes du VIII Congrès de l'Association Guillaume Budé, Paris 510 avril 1968 (Paris, 1969 [1970]), p. 67-86 (= Opera minora selecta IV, p. 383-403 ; Choix d'écrits, Paris, 2007, p. 157-171).

\section{RÉSUMÉS}

Dans le vers 491 de l'Hymne homérique à Déméter, la mention d'un lieu de culte à la divinité a récemment fait l'objet d'une proposition de correction, qui, arguant des réalités archéologiques, remplace l'obscure cité de Thessalie 'A $v \tau \rho \omega v$ par la célèbre 'A $v \delta \rho \circ$. La présente note met en doute cette correction comme impossible à la fois pour la paléographie, la métrique et la syntaxe. An emendation was recently proposed to verse 491 of the Homeric Hymn to Demeter. On the basis of archaeological finds, the place of worship of the deity in'A $v \tau \rho \omega v, \quad$ an obscure Thessalian city, was replaced by the famous'A $v \delta \rho o \varsigma$. This note questions the validity of this correction, finding it impossible in palaeographic, metrical, and syntactic terms.

\section{AUTEUR \\ DENIS ROUSSET \\ École Pratique des Hautes Études - Paris \\ denis.rousset@ephe.psl.eu}

